Archived version from NCDOCKS Institutional Repository http://libres.uncg.edu/ir/asu/

\title{
Appalachỉan
}

$\overline{\text { B O O N E, NORT H CAROLIN A }}$

\section{Does Cognitive Behavioural Therapy In The Context Of A Rural School Mental Health Programme Have An Impact On Academic Outcomes?}

\author{
By:Kurt D. Michael, John Paul Jameson, Abby Albright, Rafaella Sale, Cameron Massey, Alex \\ Kirk \& Theresa Egan
}

\begin{abstract}
Given the prevalence of mental health difficulties among children and adolescents, schools have become a suitable context for providing psychological services to those who may otherwise go untreated. The cooccurrence of mental health impairments and academic problems has been widely cited, and many school mental health (SMH) programmes have begun to assess academic variables in treatment outcome. The current study evaluated a rural SMH programme's success at treating symptoms of psychological distress among high school participants and the potential association with participants' attendance, grade point averages (GPAs) and discipline referrals. Participants were 58 high school students between 14 and 18 years old. Results indicate that non-manualized cognitive behavioural therapy administered for an average of 14.88 sessions was successful at treating mental health concerns, as measured by a selfreport questionnaire collected several times throughout treatment. In addition, a large proportion of students demonstrated improvements or stability in attendance and discipline when comparing baseline and post-treatment data, though no differences emerged between the time points for the sample as a whole. Although the findings regarding academic outcomes were not impressive overall, individual outcomes varied widely, with slightly over half of the students recording higher GPAs than at baseline. Furthermore, the fact that stability rates across the variables were reasonably high might suggest that we consider widening the definition of success to include protection from decline, reserving expectations for improvement only for those who present with difficulties in that particular academic outcome. The findings documented here illustrate the need for more nuanced approaches to understanding the relationship between intervention and academic performance.
\end{abstract}

Kurt D. Michael, John Paul Jameson, Abby Albright, Rafaella Sale , Cameron Massey, Alex Kirk \& Theresa Egan (2013) "Does Cognitive Behavioural Therapy In The Context Of A Rural School Mental Health Programme Have An Impact On Academic Outcomes? Advances in School Mental Health Promotion \#6 pp. 247-262. Version of Record Available From www.tandfonline.com 


\section{Introduction}

It is well documented that the prevalence of child and adolescent psychopathology is high, with one in five youth experiencing a diagnosable disorder during any given year (Merikangas et al., 2010; Satcher, 1999). Despite the prevalence of mental health conditions among children and adolescents in rural areas, many do not seek treatment due to a variety of barriers, including but not limited to transportation difficulties, financial burdens and the stigma associated with receiving mental health care (Owens, Watabe, \& Michael, 2011). Providing mental health services within the school context has received a great deal of attention in recent years, given that schools are a convenient point of access for students with untreated mental health concerns and reduce many common barriers to 
treatment seeking (Michael, Renkert, Wandler, \& Stamey, 2009; Owens, Murphy, Richerson, Girio, \& Himawan, 2008; Owens et al., 2011; Zirkelback \& Reece, 2010).

Reaching children and adolescents in schools who may otherwise not receive adequate mental health treatment is an important public health priority, as youth who develop mental illness at an early age portend a more severe and chronic course of symptoms when compared to their adult counterparts whose conditions emerge later in life (Satcher, 1999). Youth who experience symptoms of mental illness are at an increased risk for struggles in other areas of daily life, such as peer isolation, social difficulties and family conflict, and academic problems, such as poor attendance, discipline problems, lower academic achievement and dropout (e.g. Balfanz, Herzog, \& Mc Iver, 2007; Masten et al., 2005; Valdez, Lambert, \& Ialongo, 2011). Thus, given the relationship between mental health and these important educational and social factors, school mental health $(\mathrm{SMH})$ programmes should be designed to have as broad an impact as possible. Addressing the academic outcomes in SMH programmes is especially important to educators in the spirit of providing effective, relevant and sustainable services for youth in schools.

Indeed, there is an evidence linking early signs of mental illness with adverse academic outcomes. For example, Valdez et al. (2011) assert that early evidence of emotional and behavioural problems, such as aggression, internalizing symptoms and poor social interactions, that might indicate underlying mental illness often co-occur with academic difficulties. Similarly, Masten et al. (2005) conducted a longitudinal study with 205 youth and reported that early externalizing behaviours in young children (e.g. aggression) were found to be associated with reduced academic success by adolescence, which, in turn, appeared correlated with internalizing problems later in the teenage years and young adulthood.

As mental health difficulties and academic struggles are often seen at an early age (Valdez et al., 2011) and are interrelated throughout development (Masten et al., 2005), intervening 'in any one domain therefore may become amplified and have system-wide reverberations' (Thelen, 1989, p. 94). Based on the theorized bidirectional association between mental health impairments and academic difficulties, it stands to reason that successfully intervening in one domain would lead to positive changes in the other (Kohlberg, LaCrosse, \& Ricks, 1972; Masten et al., 2005). As researchers have demonstrated that early indicators of adjustment and functionality may foretell later adult adjustment, SMH programmes offer a unique portal into improving youngsters' wellbeing in both psychological and academic domains across the lifespan.

\section{Evaluation of SMH programmes and academic outcomes}

The importance of academic functioning as an outcome related to SMH service utilization is not, for lack of a better word, simply an academic outcome. Although SMH programmes typically evaluate success in terms of improvements in mental health functioning (e.g. Albright et al., 2013), assessing programme impact on academic outcomes is important for community stakeholders and school administration, often gatekeepers to SMH implementation and dissemination (Flaspohler, Anderson-Butcher, Paternite, Weist, \& Wandersman, 2006). However, attempts to demonstrate the impact of SMH programmes on academic outcomes have been somewhat unclear. Baskin, Slaten, Sorenson, Glover-Russell, and Merson (2010) conducted a meta-analysis of 83 studies assessing the impact of school-based psychotherapy and found a medium effect size (ES) for mental health outcomes $(\mathrm{ES}=0.50)$ and relatively smaller effect for academic 
outcomes $(E S=0.38)$. Not only were the academic outcomes more modest, there were fewer in number relative to the mental health outcomes and quite variable in terms of the constructs they were designed to measure. For instance, 'environmentally related outcomes' included attendance and discipline referrals. Furthermore, self-reported outcomes (academic self-efficacy) were of a higher magnitude $(\mathrm{ES}=0.59)$ than teacherreported outcomes (e.g. on task behaviour; $\mathrm{ES}=0.26$; Baskin et al., 2010).

The findings on the effects of SMH services on academic performance have been even more ambiguous in other studies. Sander, Everts, and Johnson (2011) reported a link between higher family involvement, higher dosage of services and increased standardized math scores at follow-up, but the standardized scores for the overall sample declined somewhat. That is, in a matched comparison between students who received services and those who did not, standardized reading scores were significantly higher among the no-treatment comparison group during one particular school year. However, the differences in reading test scores were no longer significantly different between the comparison and treatment groups at the next follow-up assessment (Everts, 2011). In other words, the authors suggested that the treatment group closed the gap between these years, though it is not clear whether intervening variables unrelated to treatment may have played a role.

Others have suggested that though improvement is the most desirable academic outcome, credence should be given to the prevention of worsening academic performance as well. There is good evidence that promotes the idea that a stabilization of grade point average (GPA) should be considered a viable treatment gain (Owens et al., 2008). In one treatment intervention that examined disruptive problem behaviours in elementary children, quarterly GPA was collected in addition to teacher and parent reports regarding emotional functioning and externalizing symptoms. Although the treatment group did not show significant grade improvement post-intervention, participants in the wait list control group exhibited a negative trajectory of worsening grades over time. The difference between grade score trends of the treatment group and control group was significant. In addition, similar results have been found in an adolescent attention-deficit/hyperactivity disorder (ADHD)-specific treatment programme (Evans, Schultz, DeMars, \& Davis, 2011). It is possible that psychological intervention for youth may act as a safeguard from academic decline in the latter years of schooling. Therefore, an unchanged GPA may be conceptualized as a positive treatment outcome.

Increasingly, researchers have chosen to include discipline-related variables under the umbrella of academic outcomes as well. Sander et al. (2011) indicated that along with improvements in teacher and parent ratings of emotional and behavioural symptoms among those youth who received SMH services, there was an associated slight decline in the number of suspensions, albeit several months after the intervention took place. Given the uneven results of past research, more research is needed to further study the relationship between SMH services and academic performance. The current study aims to contribute to the existing literature by examining the available academic outcomes among students who received mental health services during the 2011-2012 academic year. Academic performance (i.e. attendance, GPA, discipline referrals) of participants receiving services through the Assessment, Support, and Counseling (ASC) Center, a broad-based SMH programme in the rural Appalachian region of Western North Carolina, was compared at baseline, post-treatment and after a 6-month follow-up period. In addition, we examined the sample distributions of outcomes to help interpret the data. 


\section{Methods \\ Participants}

The eligible participants for this study were from a rural high school in Western North Carolina. A total of 64 students consented to receiving services through the ASC Center during the 2011-2012 academic year. Fifty-eight students had at least two administrations of the Youth Outcome Questionnaire (YOQ-30), the minimum necessary to measure pretest and post-test changes in mental health symptoms, and thus were included in the analyses. The high school was located in rural Western North Carolina, in a county with a population of approximately 51,000 according to the 2010 Census Bureau. The participants ranged from 9th to 12th graders aged between 14 and 18 years. The sample had a mean age of 16 years and 3 months [standard deviation $(\mathrm{SD})=1.19$ ]. The majority of the sample $(91 \%)$ was Caucasian, 5\% African American and 3\% Latino/ Latina. Sixty-six per cent (i.e. 38 out of 58 participants) of the sample were female. Of those participants who were seniors during the 2011-2012 school year, $81.8 \%$ (i.e. 9 out of 11 students) graduated from Watauga High School (WHS) at the end of the school year. Comparatively, WHS had a school-wide graduation rate of $85.4 \%$ at the end of the $2011-$ 2012 school year. In addition, two sophomores in the study withdrew from school enrolment during the 2011-2012 school year. Seven additional students withdrew from WHS at the beginning of the fall 2012 (follow-up) semester.

\section{Measures}

\section{Academic variables}

The academic outcomes measured in this study included GPA, attendance and discipline referrals at baseline (semester prior to receiving services, if available), the end of treatment and after a 6-month follow-up. The academic variables of interest were selected based on findings that early indicators of behavioural engagement (i.e. attendance) serve as early predictors to later academic success, even graduation from high school (Balfanz et al., 2007).

GPAs were calculated for each student's semester prior to starting services (baseline), at the end of the 2011-2012 school year (post-treatment) and 6 months later (follow-up). GPAs were calculated by dividing the number of unweighted credits (range 0.0-4.0) received by the number of classes that were taken in that semester. A typical student course load was approximately four classes per semester.

Attendance was measured by the percentage of time present during each semester of interest. When a student was absent for three or more class meetings on one school day, it was counted as one full missed day or six instructional hours missed. The percentage of attended instructional time was calculated by dividing the total attended days by 90 , which is the total number of days per semester (total semester days may vary slightly depending on the number of days missed due to inclement weather). However, if the student began services after 1 October of their year of enrolment, the number of attended days was divided by the number of days leading up to 1 October. This day was established as a cutoff point for the academic variable baseline as the same semester as the initial referral in order to increase the number of available baseline data for those students who were enrolled in another school during the previous school year. In order to capture the attendance and discipline behaviour that most closely preceded an ASC Center referral, the 1 October cut-off for baseline was used for all mid-semester ASC Center referrals, regardless of the student's location during the previous school year. 
Discipline referrals were measured by hand-counting the number of referrals given to each student involved in services at baseline, after each semester of service attainment within the school years of interest, and at a 6-month follow-up. In order to measure status at subsequent time points, the discipline data were categorized in accordance to the procedures previously utilized by Walker, Kerns, Lyon, Bruns, and Cosgrove (2010). That is, those students who received no discipline incidents in a given period were assigned a ' 0 ', those who received one discipline referral were assigned a ' 1 ' and those who received two or more referrals in a given period were assigned a ' 2 '. Discipline incidents included each individual incident that a teacher or administrator recorded or gave a referral for during the semester of interest, whether or not disciplinary action beyond the written referral was given. Although referrals related to tardiness were considered, they were not included in the analyses given the inconsistent enforcement and recording of these events during the 2011-2012 year.

\section{YOQ-30}

To assess a student's response to the ASC Center services as it pertains to mental health outcomes, the YOQ-30 (Burlingame et al., 2004) was administered at baseline (i.e. the time of intake) and at the beginning of at least every other session with the students. The items (based on a $0-4$ scale, with 0 indicating never experiencing the item and 4 indicating always, or almost always, experiencing the item) are based on a 7-day time frame and address psychological concerns and symptoms across problem types and disorders (e.g. mood and anxiety disorders, conduct and attention problems, interpersonal concerns). The YOQ-30 is a self-report measure that purports to measure treatment effects for adolescents engaged in psychotherapy. It has been found to be sensitive to changes that take place over the course of treatment. The YOQ-30 has high internal consistency, with community normative sample yielding a Cronbach's $\alpha=0.92$, and outpatient normative sample yielding a value of 0.93 (Burlingame et al., 2004). A total raw score of 29 for YOQ-30 has been identified as an appropriate cut-off in distinguishing between clinical and nonclinical levels of symptomology (Burlingame et al., 2004). Although subscales are available for scoring, the total score is the most sensitive in tracking change and has the highest reliability and validity standards.

\section{$B A S C-2$}

The Behavior Assessment System for Children - 2nd edition (BASC-2; Reynolds \& Kamphaus, 2004) is a multi-observer measure of behavioural, emotional and adaptive functioning in young people. The self-report version of the BASC-2 contains narrow and broadband scales that provide an indication of an individual's level of functioning in that particular domain. Some of the questions are rated on a dichotomous true or false format, whereas other questions are rated on a four-point Likert scale of never, sometimes, often or almost always true of that individual. The BASC-2 was administered at the time of intake and was used as a diagnostic indicator. Although no formal diagnoses were made through the ASC Center, the BASC-2 was used to assist clinicians in formulating hypotheses regarding participants' clinical presentation and problem areas. That is, the student was said to present with a significant concern on clinical scales that were elevated at least two SDs above the mean $(T$-score $>70)$.

The BASC-2 has been tested for use in the target population with a sample of children across the USA who were representative of the normal population in terms of 
'socioeconomic status, ethnicity, geographic region, and classification into specialeducation or gifted programs' (Reynolds \& Kamphaus, 2004, p. 115). The Self-Report Profile (SRP) version of the BASC-2 exhibits high internal consistency on composite scales, with $\alpha$ coefficients ranging from 0.84 to 0.96 . The SRP's test-retest reliabilities for the composite scales range from the upper 0.70 s to the lower $0.80 \mathrm{~s}$, with an interval of 13 to 66 days in-between administrations (Reynolds \& Kamphaus, 2004).

\section{Procedures}

The vast majority of students were referred to ASC Center services by school guidance counsellors or teachers; a much smaller proportion of students were self-referred or were referred by parents or other administrators. Students and their families who elected to receive ASC Center services provided full informed consent and assent to participate in treatment as well as an additional consent and assent procedure to participate in the empirical project. Approval was granted by Appalachian State University's Institutional Review Board on 21 April 2011. Each student received weekly individual therapy. The type of treatment provided was primarily non-manualized, cognitive behavioural therapy (CBT) coupled with crisis and case management as deemed appropriate for each individual case. CBT treatment was tailored for each student and in consultation with each therapist's clinical supervisor (71\% of the cases were supervised by a licensed psychologist) with a predominant CBT orientation. The treatment elements included but were not limited to psychoeducation, mood monitoring, identification of cognitive distortions, cognitive restructuring, behavioural activation, activity scheduling, exposure, relaxation training, problem-solving, social skills training, communication skills training and self-monitoring procedures. These components are consistent with the most common elements for the modularized treatment of internalizing symptoms for children and adolescents (Chorpita, Daleiden, \& Weisz, 2005). The end of spring 2012 semester was classified as the end of treatment or post-test, as the majority of students terminated services at the end of the school year. Among those students who resumed services in the 2012-2013 school year, there was a 3-month break in treatment through the ASC Center, and thus using the end of the school year as the post-test was deemed appropriate.

Eight clinicians provided individual therapy to the 58 participants. The clinicians included four graduate students in Clinical Health Psychology, one graduate student in Clinical Social Work, one graduate student in Marriage and Family Therapy, one master's level psychologist and one Licensed Clinical School Social Worker. Clinicians with psychological training provided treatment to $71 \%$ of the sample, social work clinicians provided treatment to $16 \%$ of the sample, and the Marriage and Family Therapy trained clinician met with $13 \%$ of the total sample. In addition to $1 \mathrm{~h}$ of weekly individual supervision, the therapists met weekly for an average of $2 \mathrm{~h}$ and received group supervision and consultation from the ASC team, which included three doctoral-level, licensed faculty (psychologist, clinical social worker, marriage and family therapist). Moreover, additional supervision was provided as needed when students experienced crises or when case management needs arose.

\section{Analyses}

A repeated measures ANOVA was used to compare attendance across the three time periods using only participants with complete data $(n=31)$. In addition, attendance was 
analysed in terms of the percentage of students who improved, remained stable or worsened their academic performance from baseline to post-treatment. A change (i.e. improvement or reduction) in attendance was defined as an increase or decrease in at least one-half of a SD (i.e. $4.5 \%$ ) in per cent attendance from baseline to post-treatment and follow-up semesters, or approximate increase or decrease in change of 4.05 days (24.3 instructional hours). Stability or 'no change' in attendance was defined as post-treatment and follow-up semester per cent attendance within $4.5 \%$ of the individual's baseline percentage. The rationale for this approach is based on the already high level of attendance seen among the sample at baseline; more stringent standards would exclude a large proportion of the sample due to a ceiling effect.

To aid in the interpretation of GPA data, a distribution of differences between baseline, post-treatment and follow-up time points was calculated. In addition, a repeated measures ANOVA was used to compare GPA across the three time periods. As described above, discipline incidents were categorized consistent with Walker et al. (2010). Furthermore, an improvement in functioning as measured by discipline was a reduction of referrals. That is, if a student began his or her baseline semester with ' 1 ' or ' 2 ' for discipline incidents, and improvement was if the student who began with ' 1 ' ended with ' 0 ' or the student who began with ' 2 ' ended with ' 1 ' or ' 0 '. Stabilization in discipline referrals was defined as remaining the same from baseline to post-treatment and follow-up semesters. A worsening was defined as the student who began with ' 0 ' ending with ' 1 ' or ' 2 ' at post-treatment or follow-up semesters, or the student who began with ' 1 ' at baseline ending with ' 2 ' at post-treatment or follow-up semesters. Differences in proportions across the different time points were compared using $\chi^{2}$ analyses.

To measure pre- to post-treatment changes in mental health functioning among participants, a mean difference ES within the group was computed. To measure whether the sample's changes in YOQ-30 scores from pre- to post-treatment were reliable and clinically meaningful, the Reliable Change Index (RCI) was used (Jacobson \& Truax, 1991). The RCI is a method for determining whether meaningful change has occurred following treatment by utilizing a two-part criterion. First, the client must begin treatment with symptoms that meet or exceed clinical cut-offs and end treatment in the subclinical range (Jacobson \& Truax, 1991). On the YOQ-30, a total score of 29 or higher has been established as the clinical cut-off (Burlingame et al., 2004).

The second part of the two-part criterion is that the amount of change must be sufficient enough to suggest that a reliable amount of change in symptoms has occurred, as opposed to the random fluctuation of symptoms or measurement error (Jacobson \& Truax, 1991). The RCI is the difference between individual's pre- and post-test scores and is adjusted for the standard error of the difference between the scores. In the case of the YOQ, the amount of change between baseline and post-treatment assessments to qualify for a reliable change in 10 points (Burlingame et al., 2004). Individuals who meet both criteria, meaning that they began treatment at clinically significant levels of symptoms, evidenced a decrease in their symptoms by 10 points or more, and ended treatment in the subclinical range, are considered 'recovered'. There are also several delineations of the 'non-recovered' participants. Those who exhibit the reliable change but do not move from the clinical to subclinical range are considered 'improved', those who meet neither criteria are 'unchanged' and for those whose symptoms worsen by a reliable amount are considered 'deteriorated' (Jacobson, Follette, \& Revenstorf, 1984; Jacobson \& Truax, 1991). 


\section{Results}

\section{Treatment and mental health outcomes}

For a more detailed description of the treatment protocol and mental health outcomes, see Albright et al. (2013). Thirty-one per cent of the sample presented for treatment with zero clinically significant $(T$ score $>70$ ) clinical scales on the BASC-2. Twenty-one per cent had one clinically significant clinical scale elevation, and $48 \%$ had two or more clinically significant clinical scales on the BASC-2. See Table 1 for the sample's primary and comorbid clinical scale elevations. The mean baseline YOQ-30 score for all individuals that qualified for the study $(n=58)$ was 36.97 ( $\mathrm{SD}=18.71)$. The overall sample was served for an average of $14.88(\mathrm{SD}=8.56)$ sessions or approximately $40.8 \mathrm{~min}$ per session.

The sample $(n=58)$ presented for treatment with an average YOQ-30 total score of 36.97 ( $\mathrm{SD}=18.71)$, which is in the clinically significant range (Burlingame et al., 2004). At final assessment, the entire sample $(n=58)$ had an average YOQ-30 total score of 23.31 $(\mathrm{SD}=20.07)$, which fell below the clinically significant cut-off $(>29)$. The mean difference ES between the total sample's average baseline and final YOQ-30 score was $0.70(95 \%$ $\mathrm{CI}=0.32-1.07)$.

Sixty-six per cent of the sample presented for treatment with clinically significant symptoms as measured by the baseline YOQ-30. At final assessment, $45 \%$ of those who began above the clinical cut-off were considered 'recovered', based on the RCI (Jacobson $\&$ Truax, 1991). Eighteen per cent of those who began above the clinical cut-off exhibited reliable change in symptoms, but remained above the clinical cut-off at final assessment (i.e. improved; Jacobson \& Truax, 1991). Thirty-two per cent of those who were above the clinical cut-off at baseline were considered unchanged at final assessment, or did not exhibit a reliable change in symptoms from baseline to post-treatment. Of those who began treatment above the clinical cut-off, 5\% were deteriorated at post-treatment or increased their total YOQ-30 score by 10 points or more. In addition, among the 20 students who began treatment below the clinical cut-off, three students demonstrated at least a 10-point increase in the YOQ-30 at final assessment and ended treatment above the clinical cut-off, and thus were also said to have deteriorated (Jacobson et al., 1984; Jacobson, Roberts, Berns, \& McGlinchey, 1999; Jacobson \& Truax, 1991).

\section{Academic outcomes}

\section{Grade point average}

Histograms detailing changes in GPA between baseline and post-treatment and baseline and follow-up are presented in Figure 1. Overall GPA did not significantly differ across the

Table 1. Clinically significant elevations on the BASC-2 at baseline.

\begin{tabular}{lcc}
\hline Clinical scale & Primary presenting problem (\%) & Comorbid condition (\%) \\
\hline Anxiety & 13.59 & 17.65 \\
Atypicality & 13.59 & 2.94 \\
Hyperactivity & 13.59 & - \\
Somatization & 13.59 & 17.65 \\
Attention problems & 10.81 & 20.59 \\
Locus of control & 10.81 & 2.94 \\
Sense of inadequacy & 10.81 & 5.88 \\
Depression & 5.41 & 23.53 \\
Social stress & 5.41 & 8.82 \\
Sensation seeking & 2.70 & - \\
\hline
\end{tabular}



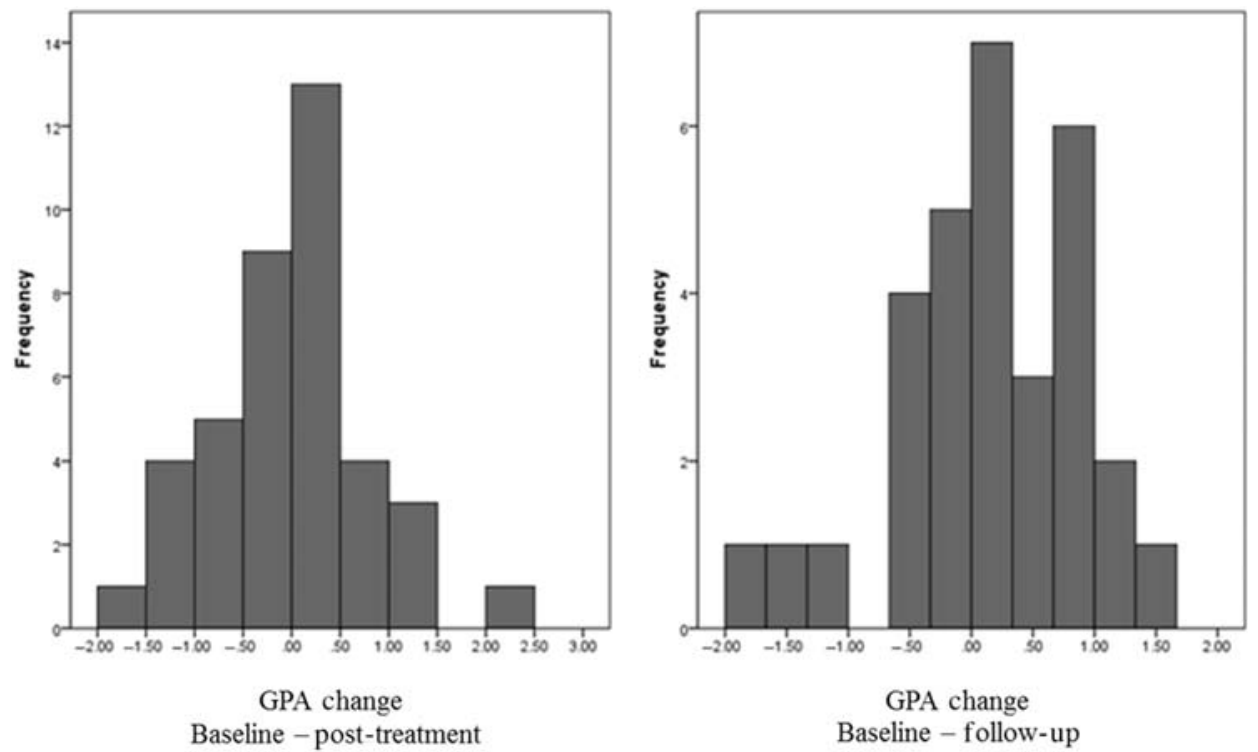

Figure 1. Distribution of GPA changes from baseline to post-treatment and baseline to follow-up.

three time periods, $F(2,66)=0.21, p=0.98$ and $\eta^{2}=0.01$. At baseline, the sample presented with an average GPA of $2.55(\mathrm{SD}=0.96)$. The sample ended treatment with an average GPA of $2.54(\mathrm{SD}=1.00)$. At follow-up, the sample had an average GPA of $2.52(\mathrm{SD}=1.05)$. Comparatively, the school-wide average GPA of WHS during the 2011-2012 school year was $3.05(\mathrm{SD}=0.71)$.

\section{Attendance}

See Table 2 for a summary of the sample's improvement, stability and reduction of per cent attendance at final and follow-up semesters compared to baseline. Attendance rates were not significantly different across the three time periods, $F(2,60)=0.476, p=0.62$ and $\eta^{2}=0.02$. At baseline, the sample was present for an average of $94 \%(\mathrm{SD}=5.7)$ or 84.6 days in a typical 90-day semester. At post-treatment, the sample was present for an average of $92.6 \%$ ( $\mathrm{SD}=5.9$ ) or 83.3 days in a 90 -day semester. At follow-up, the sample was present for an average of $94.3 \%$ ( $S D=4.7$ ) of instructional time or 84.9 days in a 90-day semester. Comparatively, WHS had an average attendance of $95.6 \%$ in the 2011-2012 school year or approximately 86 days in a 90-day semester.

In addition, changes in attendance rates were calculated to examine variability in attendance within the sample consistent with the analytic plan outlined above. Nineteen per cent of the sample improved (i.e. increased by half of an SD) their attendance at posttest. Fifty-four per cent of the sample remained stable (i.e. remained within a half of an $\mathrm{SD})$ in their per cent attendance at post-test compared to baseline semester. Those participants who either improved or remained stable in attendance were present for an average of 18.84 more instructional hours at post-test than at baseline.

Nineteen per cent of the sample improved their attendance from baseline to follow-up. Fifty-eight per cent of the sample remained stable in their per cent attendance at follow-up compared to baseline semester. Twenty-three per cent of the sample was present for at least a half of an SD less instructional time during the follow-up semester when compared 
Table 2. The total sample's per cent of improvement, stability or worsening in attendance and discipline referrals at post-treatment and follow-up semesters.

\begin{tabular}{lrr}
\hline & Post-treatment $(n=48)$ & Follow-up $(n=31)$ \\
\hline Attendance & & \\
Improved & $9(19 \%)$ & $6(19 \%)$ \\
Stable & $26(54 \%)$ & $18(58 \%)$ \\
Worse & $13(27 \%)$ & $7(23 \%)$ \\
Discipline & & \\
Improved & $8(17 \%)$ & $6(19 \%)$ \\
Stable & $29(60 \%)$ & $16(52 \%)$ \\
Worse & $11(23 \%)$ & $9(29 \%)$ \\
\hline
\end{tabular}

to baseline attendance. Those who improved or remained stable in their attendance were present for an average of 18.36 more hours at follow-up than baseline semester.

\section{Discipline events}

See Table 2 for a summary of the sample's improvement, stability and worsening of discipline events at final and follow-up semesters compared to baseline. At baseline, $29.2 \%$ of the sample with baseline data had one or more recorded discipline incidents. At post-treatment, $37.5 \%$ of the sample had at least one discipline referral. At follow-up, $38.7 \%$ of those who were present during the follow-up semester had one or more discipline referrals.

At post-treatment using the categories as described by Walker et al. (2010), 17\% of the sample improved in the number of discipline referrals by one incident or more compared to baseline semester. Sixty per cent of the sample remained stable in their discipline incidents at post-treatment compared to baseline semester, and $23 \%$ had at least one more discipline referral at post-treatment, thereby considered worse when compared to baseline. Bowker's test for symmetry revealed no significant differences across the three categories at post-treatment, $\chi^{2}(3, N=48)=2.81, p=0.42$. At follow-up, $19 \%$ of the sample who were still enrolled in school improved the number of discipline referrals by one incident or more compared to baseline semester. Fifty-two per cent of the sample remained stable in their discipline referrals at follow-up compared to baseline, and $29 \%$ of the sample had at least one more discipline referral at follow-up semester and considered worse when compared to baseline. Bowker's test for symmetry revealed no significant differences across the three categories, $\chi^{2}(3, N=31)=2.20, p=0.53$.

\section{Discussion}

The results of the study indicate that an average of approximately 15 sessions of individual CBT provided in the context of a broad SMH programme was associated with significant improvements in psychological functioning for the majority of those who underwent treatment. Almost two-thirds of those who began treatment in the clinical range decreased their symptoms by a reliable amount. As a group, the mean YOQ-30 score was below the clinical cut-off and in the normative range of psychological functioning at the final assessment.

Overall differences in attendance, GPA or discipline referrals between time points did not emerge, but a substantial proportion of participants remained stable or improved over time, while a lesser proportion worsened in these areas (dramatically in some cases). 
Slightly more than half of the sample had higher GPAs at post-treatment compared to baseline, and more than $60 \%$ had higher GPAs at follow-up compared to baseline. We also note that the sample variance in GPA was higher at each time point than GPA variance for the entire student body. Though this evidence is by no means definitive, it may suggest pronounced differential response in academic achievement as a result of participation in SMH interventions, where many of the participants stabilize and improve. That said, we recognize that GPA for the treated sample was significantly lower than the mean GPA for the school as a whole, and that a significant proportion did not show improvement or stability in GPA at post-treatment or follow-up.

The findings also suggest that though no differences were found between time points in mean attendance rates, roughly three-quarters of the sample remained stable or improved at post-treatment and follow-up. In keeping with the stability theme, the post-treatment attendance numbers were reasonably strong for the sample. In addition, given that the attendance rates of the treated students were both very high and very similar to the rates for the school as a whole (94\% vs. $96 \%$, respectively), any improvement in this area would have been difficult to achieve. Attendance did not appear to be a significant academic problem for students participating in SMH interventions at baseline. Moreover, protecting students from deterioration in the form of stability appears a sensible goal (Balfanz et al., 2007). This type of finding, where the maintenance of a positive trajectory is associated with treatment (e.g. Multisystemic Therapy), is construed as a beneficial effect (e.g. Brown, Henggeler, Schoenwald, Brondino, \& Pickrel, 1999). Still, among those students who were enrolled during the follow-up semester, those categorized as improved or stable in their attendance exhibited an average increase by $18 \mathrm{~h}$ in instructional time at follow-up when compared to baseline. Thus, the support for SMH programmes and the impact on academic outcomes might be reflected in the fact that attendance problems never emerged for the majority of participants. Conversely, the finding also highlights the fact that a low number of youth in the sample account for a disproportionately high number of absences. Among the students with complete data who withdrew or dropped out since the start of the 2011-2012 year, seven of the eight had baseline attendance rates (74\%) well below the school and sample means. Balfanz et al. (2007) documented that particular attendance thresholds (e.g. $<90 \%,<80 \%$ ) portend progressively higher risk for dropout. Thus, these data might suggest that SMH interventions should focus more aggressively on retention once students who exhibit these attendance patterns are identified.

Changes in discipline referrals over time followed a similar pattern to that of attendance and GPA. Roughly three-quarters showed either stabilization or improvement in discipline events across the three time points. Nonetheless, a relatively small proportion of the sample presented with discipline problems at any point during the study. This highlights an inherent limitation of using discipline referrals as a metric of academic outcome. Although it is true that a minority of students who utilized the SMH services had discipline issues, it is not applicable to most users. Therefore, an examination of discipline variable should be limited only to those students for whom it creates larger academic difficulties.

Overall, this study suggests that though the sample as a whole did not differ in measures of academic functioning over time, a majority of students did see their performance stabilize or improve while others deteriorated. The high degree of variability in these outcomes, while not uncommon (e.g. Walker et al., 2010), does indicate that if a relationship exists between SMH interventions as practiced in the community and changes in academic performance, it is likely not a simple direct effect and therefore warrants 
further investigation. Several possibilities might account for the patterns of differential response seen in this study, including demographic factors, presenting problems and treatment techniques. The type of problem that the student presents with may be related to later academic outcomes. For example, clinical depression or generalized anxiety disorder may cause more academic impairment than adjustment issues or relationship discord. Successful treatment of depression may lead to significantly improved academic functioning, while resolving relationship conflicts may have little effect on academic performance. Such differences may not be captured by general treatment outcome questionnaires like the YOQ-30. Similarly, some treatment techniques may be more relevant to classroom performance. For instance, behavioural approaches to the treatment of ADHD such as time management/activity scheduling and problem-solving skills may be directly relevant to academic performance and GPA, but relaxation training for anxiety may not be as applicable to the classroom setting.

Additional variables outside the scope of the presenting problem and treatment approach also may mediate the relationship between SMH interventions and academic outcomes. One variable that may hold particular promise is school connectedness, which has been shown to be related to both internalizing symptoms and completion of school (Bond et al., 2007; Schochet, Dadds, \& Montague, 2006). However, no known attempts to examine school connectedness as a mediator between treatment success and academic performance have been undertaken. Such studies may further elucidate the relationship between mental health and academic performance and point to ways that SMH assessment treatment strategies could be modified to better address academic functioning.

Taken together, it appears that the academic outcomes measured in this study are relevant only for a select number of students who underwent SMH treatment. This makes the study of academic outcomes somewhat problematic for SMH researchers. That is, as clinicians, we regularly individualize treatment for the elevated clinical variables (e.g. depression) and not others (e.g. ADHD) for specific students. Thus, it would not be sensible to focus on all of the academic findings unless they were significant or relevant for those students. Moreover, academic variables such as GPA, discipline variables and attendance typically are treated as universal outcomes despite the fact that many, if not most, students who use SMH services are not necessarily struggling with these issues. Just as we would not universally predict changes in depression symptoms resulting from the treatment for oppositional defiant disorder, we should not always expect changes in academic outcomes resulting from the treatment for mental distress. Instead, studies in the changes in academic outcomes should focus primarily on those we suspect are struggling academically as a result of mental or emotional distress.

\section{Limitations, clinical implications, future directions}

Although the empirical findings regarding academic outcomes, including those presented here, suggest that modest improvements in attendance, discipline and scholastic performance occurred for a proportion of students in treatment, it often requires patience and persistence to see these improvements over time. Walker et al. (2010) reported an initial dip in attendance among SBHC users, but that attendance rates actually increased at a greater rate over time and that these data were not evident until the students had been followed for a sufficient amount of time. Likewise, Sander et al. (2011) found evidence that more intense dosages of SMH treatment over time were associated with narrowing gaps in math achievement scores between recipients and matched controls during the follow-up periods. The results from this study appear to mirror the previous findings in 
some respects, but it also calls attention to some limitations. The most significant limitation of the study is the relatively short time frame from baseline to post-treatment, and post-treatment to follow-up, approximately 6 months later. More specifically, the students seen by the ASC Center were relatively high risk to begin with across the academic variables under study, especially in terms of GPA. With a longer follow-up period, the gains that were beginning to emerge after 6 months might continue on an upward trend. Thus, following the current sample longitudinally would be an appropriate future direction.

Another limitation was the inconsistent recording of GPAs. Based on the existing data management system at the school, student schedules were removed from the system at the end of the school year, making it impossible to differentiate between the fall and spring semester courses that were taken within a given school year. Consequently, in those cases $(n=11)$, full-year GPA was used instead of semester-by-semester grades. In these cases, the sensitivity to changes in GPA over time is greatly reduced.

A third limitation included the lack of procedures to track the integrity of the treatment provided by the ASC Center. Because the majority of the caseload was supervised by a licensed psychologist with a CBT focus, CBT core elements were the primary mode of intervention. However, the intervention was non-manualized and, thus, there was no strict set of session instructions for therapists to follow. The CBT components were emphasized, yet implemented in a flexible manner as appropriate to each case. The question of practicability of implementing manualized programmes in the school systems has been discussed previously (Everts, 2011) and the settings in which SMH programmes are offered were thought to restrict the feasibility of manualizing programmes.

Finally, a limitation of the study was the use of a within-subjects design. Because students served as their own controls, it was not possible to determine the extent to which the changes observed were associated with the intervention. More stringent comparisons including the effects of treatment as usual or the utilization of a no-treatment control group would have strengthened the study. In addition, attrition of students meant that the sample size for comparisons across the three time periods was significantly reduced. Furthermore, it is possible that students who did not complete follow-up assessment may have been the most vulnerable to worsening of academic and mental health outcomes given that several of these students withdrew.

The issue of utilizing a no-treatment control group brings up a specific practical barrier when attempting to implement research in school settings and potentially significant ramifications of not serving students who are in need of mental health treatment. School systems are not likely to allow researchers to provide care only to a subsection of students in distress while another subsection goes underserved, particularly when mental health problems are interfering with both the child's ability to learn and educator's ability to teach. Politically, local Boards of Education may come under fire for allowing researchers a presence in a school simply to study the children without actually providing treatment, and are likely not to approve any agreement between any potential county-university partnerships. If researchers are attempting to collect any data on the effectiveness of mental health treatment in schools, it must be done with consideration of the school and framed in such a way as to develop long-term methods for helping educators continue to focus on educating students. Due to these limitations, ongoing improvements and revisions in data collection methods should be implemented for future SMH studies. One such strategy that might be used as an alternative to control groups is benchmarking, which has been utilized to examine the effectiveness of empirically supported treatments in community settings (e.g. Wade, Treat, \& Stuart, 1998). As it applies to the assessment 
of academic performance, SMH treatment programme outcomes could be compared to control groups from randomized controlled trials conducted outside of the school setting if these future studies include assessment of academic variables. Although such designs are not without potential limitations (e.g. systematic differences between trial samples and SMH samples, differences in treatment approaches or dosage, history effects), they may provide additional clarity on the effects of SMH interventions on academic outcomes.

In addition to improving the methods of data collection, future studies in SMH may benefit from expanding academic variables to include areas that reflect a more immediate response to treatment. For instance, depending on when a student begins treatment in the semester, the student may already have too many low grades to have a chance for ongoing treatment to impact his or her GPA. Therefore, solely relying on academic 'achievement' fails to capture other critical aspects in the school setting. Broadening academic variables to include examining teachers' perceptions of the students, student engagement (e.g. participation in class, completion of in-class/homework assignments, classroom disruptions) and also considering items on the BASC (e.g. Attitude towards School, School Problems) may provide a more accurate picture of whether treatment is affecting the students' academics. Furthermore, developing a Clinical Global Improvement scale for school may also prove to be useful in depicting a more immediate impact of SMH. Going forward, it is necessary for SMH interventions to increase the academic variables that are assessed, as key factors in the school setting are currently being under-examined.

In summary, approximately 15 sessions of CBT were effective at reducing psychological distress in the majority of adolescents who took part in a rural $\mathrm{SMH}$ programme, as shown by the pre and post-treatment mean differences of reported clinical symptoms. In addition, a large proportion of students served by the ASC Center during the 2011-2012 school year demonstrated improvements or stability in attendance and discipline when comparing baseline and post-treatment data, though no differences emerged between the time points for the sample as a whole. Although the findings regarding academic outcomes were not impressive overall, individual outcomes varied widely, with slightly over half of the students recording higher GPAs than at baseline. Furthermore, the fact that stability rates across the variables were reasonably high might suggest that we consider widening the definition of success to include protection from decline, reserving expectations for improvement only for those who present with difficulties in that particular academic outcome. The findings documented here illustrate the need for more nuanced approaches to understanding the relationship between intervention and academic performance.

\section{References}

Albright, A.E., Michael, K.D., Massey, C.S., Sale, R.D., Kirk, A., \& Egan, T.E. (2013). An evaluation of an interdisciplinary rural school mental health programme in Appalachia. Advances in School Mental Health Promotion, 6, 189-202. doi:10.1080/1754730X.2013.808890

Balfanz, R., Herzog, L., \& Mac Iver, D.J. (2007). Preventing student disengagement and keeping students on the graduation path in urban middle-grades schools: Early identification and effective interventions. Educational Psychologist, 42, 223-235.

Baskin, T.W., Slaten, C.D., Sorenson, C., Glover-Russell, J., \& Merson, D.N. (2010). Does youth psychotherapy improve academically related outcomes? A meta-analysis. Journal of Counseling Psychology, 57, 290-296. doi:10.1037/a0019652

Bond, L., Butler, H., Thomas, L., Carlin, J., Glover, S., Bowes, G., \& Patton, G. (2007). Social and school connectedness in early secondary school as predictors of late teenage substance use, mental health, and academic outcomes. Journal of Adolescent Health, 40, 357.e9-357.e18.

Brown, T.L., Henggeler, S.W., Schoenwald, S.K., Brondino, M.J., \& Pickrel, S.G. (1999). Multisystemic treatment of substance abusing and dependent juvenile delinquents: Effects on 
school attendance at posttreatment and 6-month follow-up. Children's Services: Social Policy, Research, \& Practice, 2, 81-93.

Burlingame, G.M., Dunn, T., Hill, M., Cox, J., Wells, M.G., Lambert, M.J., \& Brown, G.S. (2004). Administration and scoring manual for the Y-OQ-30.2: Youth. Wilmington, DE: American Professional Credentialing Services.

Chorpita, B.F., Daleiden, E.L., \& Weisz, J.R. (2005). Identifying and selecting the common elements of evidence based interventions: A distillation and matching model. Mental Health Services Research, 7, 5-20. doi:10.1007/s11020-005-1962-6

Evans, S.W., Schultz, B.K, DeMars, C.E., \& Davis, H. (2011). Effectiveness of the Challenging Horizons after-school program for young adolescents with ADHD. Behavior Therapy, 42, $462-474$.

Everts, J.C. (2011). Minneapolis Public Schools Expanded School Mental Health program: Outcomes from the first four years (Unpublished doctoral dissertation). University of Minnesota, Minneapolis.

Flaspohler, P., Anderson-Butcher, D., Paternite, C.E., Weist, M., \& Wandersman, A. (2006). Community science and expanded school mental health: Bridging the research to practice gap to promote child well being and academic success. Educational and Child Psychology, 23, 27-41.

Jacobson, N.S., Follette, W.C., \& Revenstorf, D. (1984). Psychotherapy outcome research: Methods for reporting variability and evaluating clinical significance. Behavior Therapy, 15, 336-352.

Jacobson, N.S., Roberts, L.J., Berns, S.B., \& McGlinchey, J.B. (1999). Methods for defining and determining the clinical significance of treatment effects: Description, application, and alternatives. Journal of Consulting and Clinical Psychology, 67, 300-307.

Jacobson, N.S., \& Truax, P. (1991). Clinical significance: A statistical approach to defining meaningful change in psychotherapy research. Journal of Consulting and Clinical Psychology, $59,12-19$.

Kohlberg, L., LaCrosse, J., \& Ricks, D. (1972). The predictability of adult mental health from childhood behavior. In B.B. Wilman (Ed.), Manual of child psychopathology (pp. 1217-1284). New York, NY: McGraw-Hill.

Masten, A.S., Roisman, G.I., Long, J.D., Burt, K.B., Obradovic, J., Riley, J.R., ... Tellegen, A. (2005). Developmental cascades: Linking academic achievement and externalizing and internalizing symptoms over 20 years. Developmental Psychology, 41, 733-746.

Merikangas, K.R., He, J., Burstein, M., Swanson, S.A., Avenevoli, S., Cui, L., . . Swendsen, J. (2010). Lifetime prevalence of mental disorders in US adolescents: Results from the National Comorbidity Study-Adolescent Supplement (NCS-A). Journal of the American Academy of Child and Adolescent Psychiatry, 49, 980-989.

Michael, K.D., Renkert, L.E., Wandler, J., \& Stamey, T. (2009). Cultivating a new harvest: Rationale and preliminary results from a growing interdisciplinary rural school mental health program. Advances in School Mental Health Promotion, 2, 40-50.

Owens, J.S., Murphy, C.E., Richerson, L., Girio, E.L., \& Himawan, L.K. (2008). Science to practice in underserved communities: The effectiveness of school mental health programming. Journal of Clinical Child and Adolescent Psychology, 37, 434-447. doi:10.1080/15374410801955912

Owens, J.S., Watabe, Y., \& Michael, K.D. (2011). Culturally responsive school mental health in rural communities. In C.S. Clauss-Ehlers, Z. Serpell \& M.D. Weist (Eds.), Handbook of culturally responsive school mental health: Advancing research, training, practice, and policy (pp. 31-42). New York, NY: Springer.

Reynolds, C.R., \& Kamphaus, R.W. (2004). BASC-2: Behavioral assessment system for children manual (2nd ed.). Circle Pines, MN: AGS Publishing.

Sander, M.A., Everts, J., \& Johnson, J. (2011). Using data to inform program design and implementation and make the case for school mental health. Advances in School Mental Health Promotion, 4, 13-21.

Satcher, D. (1999). Mental health: A report of the surgeon general - Executive summary. Professional Psychology: Research and Practice, 31, 5-13.

Schochet, I.M., Dadds, M.R., \& Montague, R. (2006). School connectedness is an underemphasized parameter in adolescent mental health: Results of a community prediction study. Journal of Clinical Child and Adolescent Psychology, 35, 170-179.

Thelen, E. (1989). Self-organization in developmental processes: Can systems approach work? In M.R. Gunnar \& E. Thelen (Eds.), Systems and development: The Minnesota symposia on child psychology (Vol. 22, pp. 77-117). Hillsdale, NJ: Erlbaum. 
Valdez, C.R., Lambert, S.F., \& Ialongo, N.S. (2011). Identifying patterns of early risk for mental health and academic problems in adolescence: A longitudinal study of urban youth. Child Psychiatry and Human Development, 42, 521-538. doi:10.1007/s10578-011-0230-9

Wade, W.A., Treat, T.A., \& Stuart, G.L. (1998). Transporting an empirically supported treatment for panic disorder to a service clinic setting: A benchmarking strategy. Journal of Consulting and Clinical Psychology, 66, 231-239.

Walker, S.C., Kerns, S.E.C., Lyon, A.R., Bruns, E.J., \& Cosgrove, T.J. (2010). Impact of schoolbased health center use on academic outcomes. Journal of Adolescent Health, 46, 251-257.

Zirkelback, E.A., \& Reese, R.J. (2010). A review of psychotherapy outcome research: Considerations for school-based mental health providers. Psychology in the Schools, 47, $1084-1100$. 